\title{
A rare case of eosinophilic cystitis involving the inside and outside of the urinary bladder associated with an infected urachal cyst
}

\author{
Hyun Bin Shin ${ }^{1}$, Hyun Sik Park', Joo Heon Kim ${ }^{2}$ and Jinsung Park ${ }^{1,3^{*}}$ (D)
}

\begin{abstract}
Background: Eosinophilic cystitis is a rare inflammatory disease of the bladder characterized by eosinophilic infiltration of the bladder wall. Most Eosinophilic cystitis cases present with mucosal lesions of the urinary bladder. We present a very rare case of large mass-forming eosinophilic cystitis, involving the inside and outside of the bladder associated with an infected urachal cyst.

Case presentation: A 59-year-old man presented with gross hematuria, fever, dysuria, and suprapubic pain. Computed tomography showed a heterogeneously enhancing mass that measured $7.6 \mathrm{~cm} \times 4 \mathrm{~cm}$ located on the anterosuperior portion of the bladder with an internal fluid collection. Cystoscopy revealed a raspberry-like mass lesion on the bladder dome. Transurethral resection of the bladder was initially performed. The mass lesion protruding from inside the bladder was removed, and pus-like fluid was drained. The pathologic diagnosis was eosinophilic cystitis. Follow-up computed tomography showed a remnant mass outside the bladder and urachal cyst. To eliminate the remnant lesion, robot-assisted partial cystectomy was performed. The patient showed no evidence of recurrent disease on follow-up cystoscopy and computed tomography for up to 2 years.
\end{abstract}

Conclusions: Clinicians should consider the possibility of eosinophilic cystitis in patients who present with hematuria, fever, and suprapubic pain and have both intravesical and extravesical masses.

Keywords: Eosinophilic cystitis, Urachal cyst, Partial cystectomy

\section{Background}

Eosinophilic cystitis (EC) is a rare inflammatory disease of the bladder, characterized by eosinophilic infiltration of the bladder wall and associated with fibrosis with or without muscle necrosis [1]. Because of the small number of patients afflicted with EC, the etiology of EC is not well understood. But subsequent mast cell degranulation is thought to be due to antigenic stimulation that promotes IgE-mediated attraction of eosinophils throughout the

\footnotetext{
*Correspondence: jspark.uro@gmail.com

${ }^{3}$ Department of Urology, Uijeongbu Eulji Medical Center, Eulji University

School of Medicine, 712, Dongil-ro, Uijeongbu-si, Gyeonggi-do 11759,

Republic of Korea

Full list of author information is available at the end of the article
}

bladder wall [2-5]. The most common symptoms include urinary frequency, hematuria, dysuria, urinary retention, and suprapubic pain.

Most EC cases present with mucosal lesions of the urinary bladder $[6,7]$, although several studies have reported mass-forming or malignancy-mimicking EC [1, 2, 6-9]. To diagnose EC, pathologic diagnosis of bladder mucosal lesions such as transmural eosinophil infiltration and absence of malignant cells by transurethral resection of the bladder (TURB) or cystoscopic biopsy, is essential $[1,2,4,6,7]$. To date, there is only one case report on EC associated with infected urachal remnants [8]; but the patient was a 4-year-old child diagnosed with EC at 7 months after surgical excision of the infected urachal cyst, with recurrent abscesses in the abdomen, pelvis, 
and at the operation site. In our case report, we present a rare case of large mass-forming $\mathrm{EC}$, involving both the inside and outside of the urinary bladder associated with an infected urachal cyst.

\section{Case presentation}

A 59-year-old man presented with gross hematuria, fever $\left(37.9{ }^{\circ} \mathrm{C}\right)$, dysuria, and suprapubic pain at the Eulji University Hospital. The patient had no history of allergies or bladder injury, and had an unremarkable medical history. Laboratory tests showed elevation in c-reactive protein levels $(17.31 \mathrm{mg} / \mathrm{dL})$ but no leukocytosis $\left(7.810 \times 10^{3} /\right.$ $\mu \mathrm{L}$ ), elevation of erythrocyte sedimentation rate (ESR, $6 \mathrm{~mm} / \mathrm{h})$, and peripheral eosinophilia $(270$ cells $/ \mu \mathrm{L})$. Urinalysis revealed hematuria, but no bacterial growth was found in urine culture.

Computed tomography (CT) showed a heterogeneously enhancing mass that measured $7.6 \mathrm{~cm} \times 4 \mathrm{~cm}$ on the anterosuperior portion of the bladder (Fig. 1). The mass had a sphere-like shape and a thickened outer wall with an internal fluid collection. Initially, an infected urachal cyst was suspected because the patient had a fever, and empirical intravenous antibiotics (third-generation cephalosporin) were initiated. Cystoscopy showed a huge raspberry-like mass lesion on the bladder dome with a definite margin, which occupied more than half of the bladder (Fig. 2A, B). Because the possibility of a tumorous condition could not be fully excluded, percutaneous drainage was not performed. After infection control with intravenous antibiotics, TURB was performed for pathologic diagnosis. During resection, a pus-like fluid was found inside the mass (Fig. 2C), and most mass lesions inside the bladder were removed (Fig. 2D). Tissue and fluid culture were performed, but no bacterial growth was observed.

Histopathological examination revealed numerous eosinophilic infiltrates in the bladder wall. These were diffusely infiltrated and gathered around vessels in the lamina propria and muscularis propria. Mild urothelial papillary hyperplasia was present. No viral inclusions were observed. Immunohistochemical staining showed patchy positive reaction on CK20 and CD 44 proteins. The tumor protein P53 showed score 1 (less than 5\%) positivity. The pathologic diagnosis was EC (Fig. 3).

At 2 weeks postoperatively, follow-up CT showed shrinkage of the mass lesion, but a remnant ulcerative lesion, which was thought to be the outer part of the mass lesion, was still noted at the bladder anterior and dome side (Fig. 4A). Another 1.4-cm cystic mass, thought to be the urachal cyst, was incidentally detected above the remnant mass (Fig. 4B). Thus, the mass that was resected with a resectoscope had EC, and the infected urachal cyst existed above the mass. To eliminate the remnant EC lesion and urachal cyst,
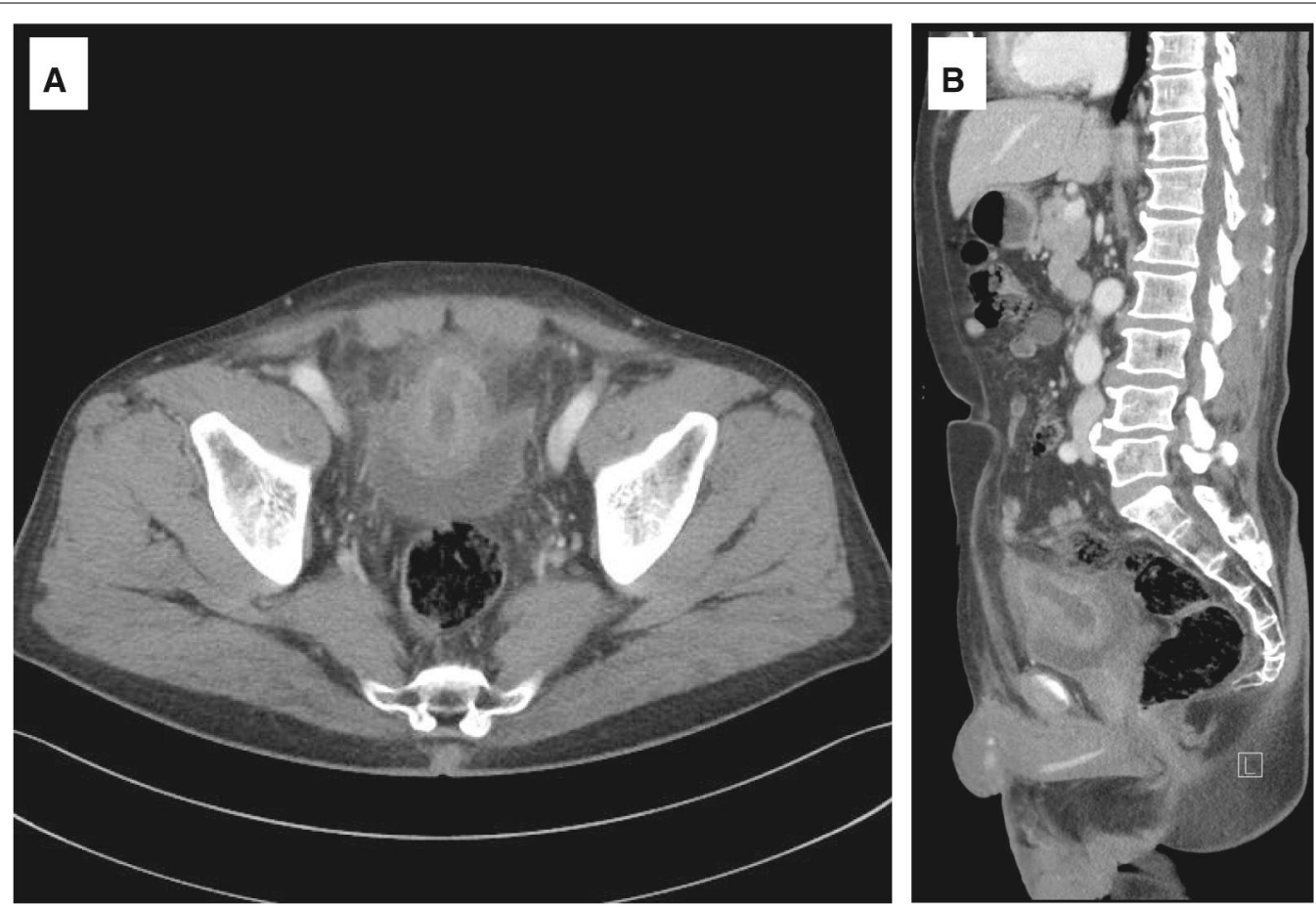

Fig. 1 Computed tomography showing a heterogeneous enhancing mass on the anterosuperior portion of the bladder. A Axial view, B sagittal view 

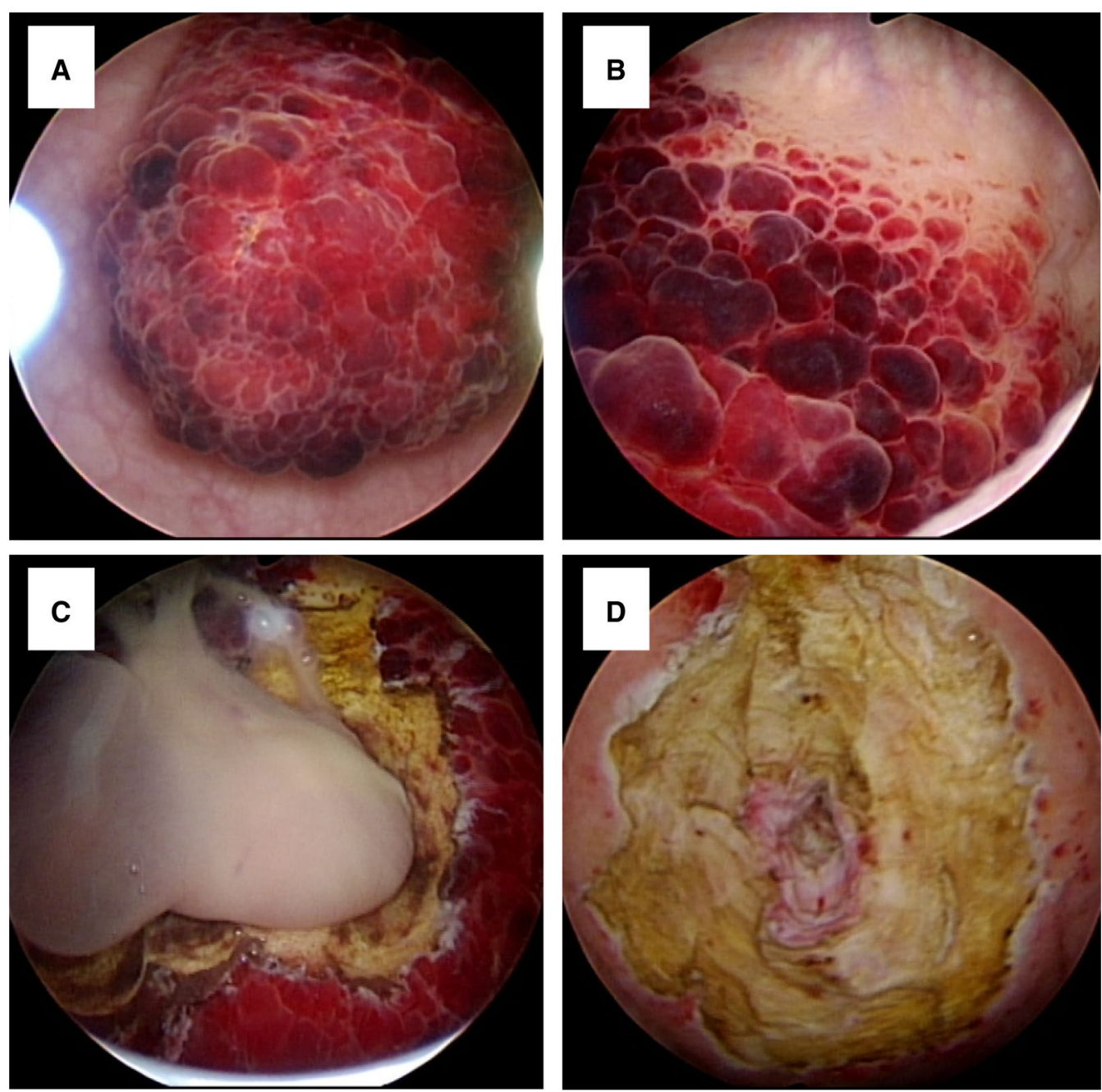

Fig. 2 Cystoscopic findings of bladder lesion. A, B Raspberry-like lesion on bladder dome. C At transurethral resection of the bladder, pus-like fluid is visible after resection of the mass lesion. D Tumor bed after mass resection. After resection of the mass lesion inside the bladder, the lesion outside the bladder is observed in the center

robot-assisted partial cystectomy was performed (Fig. 5). The urachal cyst was removed with a whole layer of the bladder wall that was, associated with the EC lesion. Similar to that seen in TURB lesions, partial cystectomy specimens showed heavy eosinophilic infiltration on the whole layers of the bladder wall, mucosal erosion, and fibrosis in the subepithelial connective tissue. An infected urachal cyst is accompanied by numerous lymphocytes, plasma cells, and histiocytic infiltrates. The benign epithelium of the urachal cyst is made up of cuboidal-type urothelium.

After partial cystectomy, the patient recovered uneventfully and was discharged on postoperative day 7 . The patient was treated with antihistamine (hydroxyzine hydrochloride) for 15 months and regularly followed up. There was no evidence of recurrence in the follow-up CT and cystoscopy for up to 23 months.

\section{Discussion and conclusion}

To the best of our knowledge, this is the first report on EC that formed mass lesions involving both the inside and outside of the urinary bladder associated with infected urachal cysts. The majority of EC cases present with erythematous bladder mucosal lesions or bullous edematous lesions without a definite cause $[4,5,7$, $10,11]$. Although a few patients have been reported to present with bladder tumor-mimicking lesions $[4,5,7$, $10,12]$, there is a case report of a pediatric patient who had a bladder tumor-mimicking lesion wherein the same was found after surgical excision of the infected urachal 

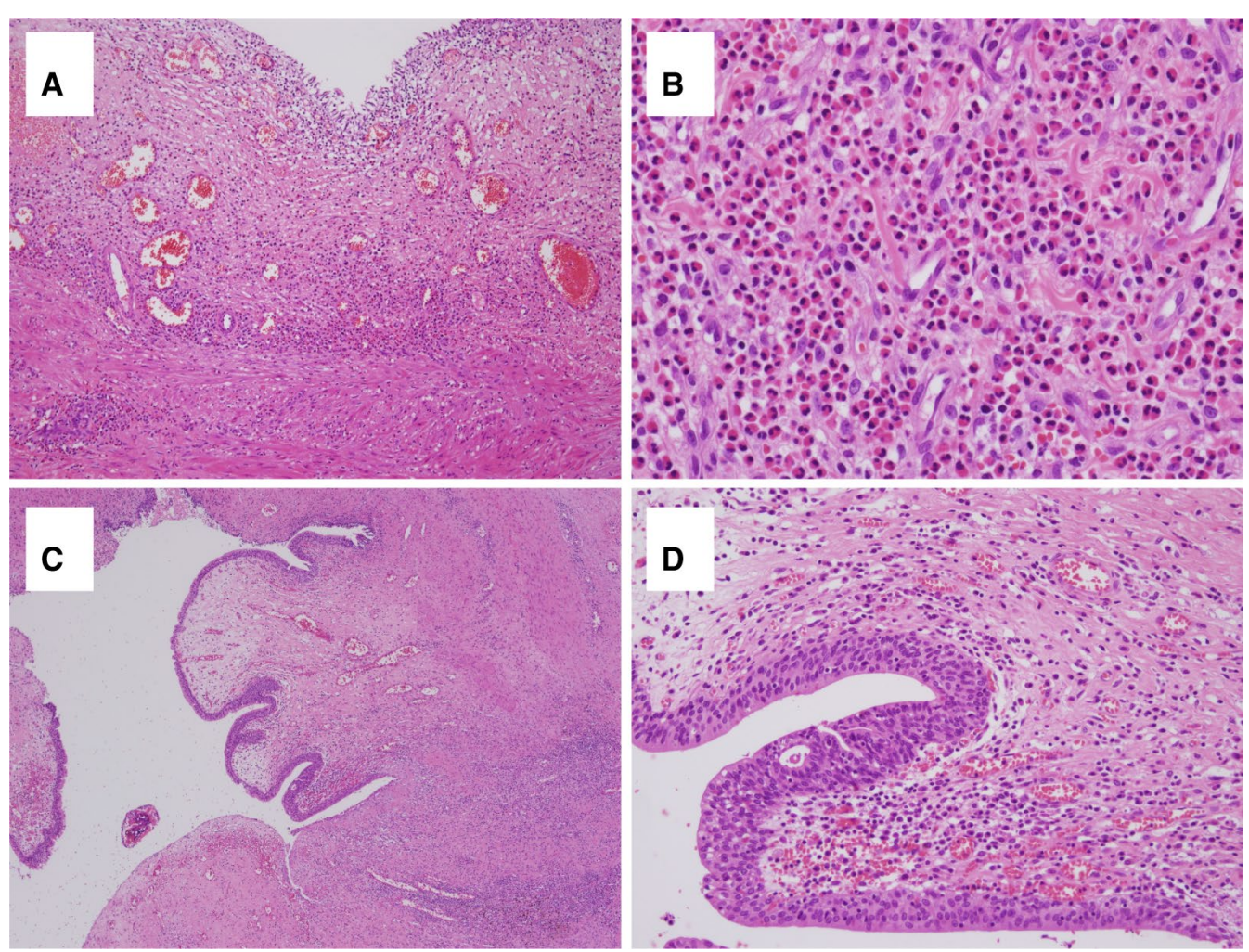

Fig. 3 Histologic findings of partial cystectomy specimen. A Low magnification photomicrography showing subepithelial and perivascular inflammatory cell infiltrate in the bladder (H\&E. $\times 100)$. B Histopathology showing many eosinophils in the bladder wall (H\&E. $\times 400)$. C The infected urachal cyst lined by urothelium and surrounded by mixed inflammatory cell infiltrate. (H\&E. X40). D The infected urachal cyst, histopathology showing numerous lymphocytes and plasma cells (H\&E. ×200)
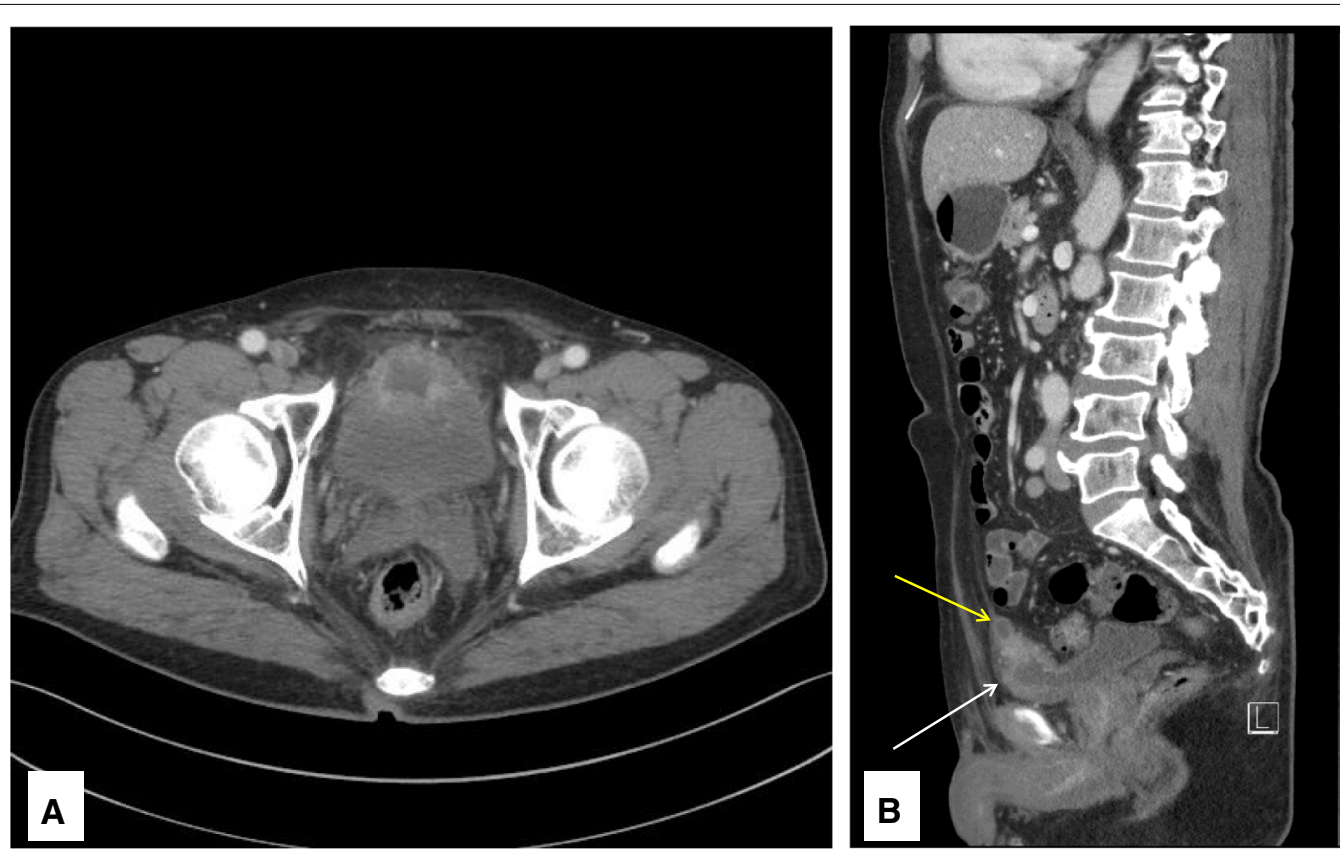

Fig. 4 Follow-up computed tomography after transurethral resection shows heterogeneous enhancing mass, reduced in size, on the anterosuperior portion of the bladder. A Axial view of bladder mass. B Sagittal view of remnant bladder mass (white arrow) with an urachal cyst (yellow arrow) 


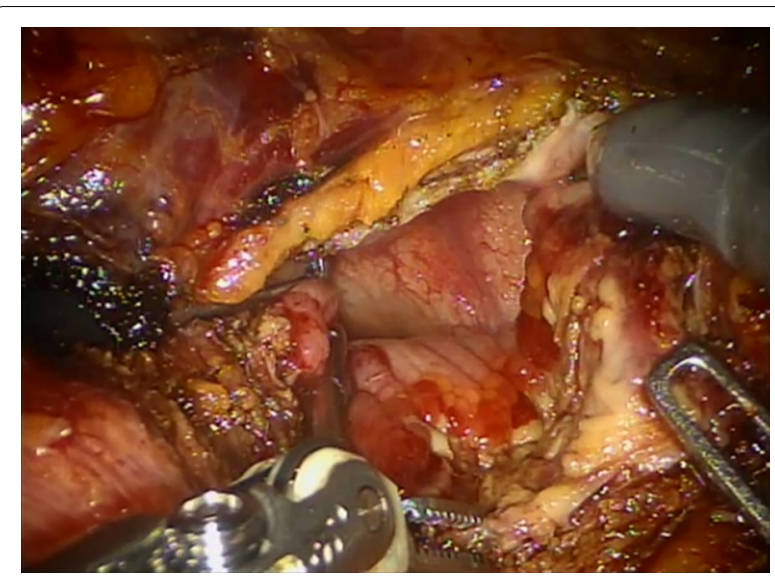

Fig. 5 Intraoperative view of robot-assisted partial cystectomy. Remnant lesion of eosinophilic cystitis after transurethral resection of the bladder (TURB) is identified at the bladder dome, i.e., remnant ulcerative, and a raspberry-like lesion is still visible. The lesion is completely removed with the infected urachal cyst, and the bladder is closed with continuous running suture

remnant [8]. In our case report, the patient presented with a sphere-like mass involving the inside and outside of the bladder as well as an infected urachal cyst. When the patient was initially presented, the bladder lesion was difficult to diagnose with EC because a huge berrylooking mass mimicking a bladder tumor was visible. To confirm the pathologic diagnosis and distinguish malignancy, TURB was performed.

Histopathological examination revealed heavy infiltration of eosinophils around vessels in the lamina propria and muscularis propria of the bladder mucosa. The diagnosis of $\mathrm{EC}$ can be made when many eosinophils are visible in bladder specimens $[1,2,4,6,7]$. There are no definite pathologic criteria for EC, but more than 25 eosinophils per high power field of view can be thought of as indicating EC, similar to eosinophilic esophagitis and gastritis [13]. In our case, heavy infiltration of eosinophils was visible around the mucosa and vessels in the lamina propria and muscularis propria, therefore diagnosis of EC could be made.

In laboratory findings, EC usually shows hematuria or proteinuria [14]. Furthermore, peripheral eosinophilia was observed in $42.5 \%$ to $>50 \%$ of the patients, and elevated ESR was observed in $7 \%$ of the patients $[1,7,14]$. In our case, the patient only showed hematopyuria without bacterial growth, and no elevation of ESR and peripheral eosinophilia was observed.

The etiology of EC is not well known because of the small number of cases. In most cases, allergies or bladder injuries are present [6]. Since EC is a result of antigen-antibody complexes $[4,5]$, avoiding antigens is also an effective method to treat EC. However, finding antigen is difficult in most cases, aside from using bladder instillation therapy. In our case, the patient had no allergies or history of bladder injury, but an infected urachal cyst was incidentally detected. Infected urachal cysts can act as allergens and attract eosinophils to the bladder wall. The infected urachal cyst may also have caused bladder injury due to the mass effect. Hypothetically, bladder inflammation induced by an infected urachal cyst could be the cause of EC. However, because the urachal cyst in our case was small (maximum diameter $1.7 \mathrm{~cm}$ ) and the patient had no specific history (allergy, medical history, or bladder injury), it is not clear whether the small urachal cyst caused EC in this patient.

There is no definite curative treatment for EC, but medical treatments such as corticosteroids, antihistamines, and antibiotics can be considered as the primary treatment [3-5, 7, 14-16]. Corticosteroids can help control inflammation and symptoms, and antihistamines can help suppress the inflammatory reaction, which inhibits the formation of immune complexes. Antibiotics can be used when EC is associated with urinary tract infection. In our case, antibiotics were initially used because the mass was thought to be related to an infected cystic lesion, and antihistamines were used to prevent recurrence after partial cystectomy.

If corticosteroids or antihistamines could not resolve EC, surgical treatment is also an option [1, 5, 7, 16-18]. Surgical treatment not only has a high treatment success rate, but also a low recurrence rate $[4,7]$. In our case, the reason for choosing partial cystectomy was that it required removal of the remnant urachal cyst. On CT, both urachal cysts and EC lesions were visible after TURB, and partial cystectomy was a good option to remove both lesions in one surgery.

Although the prognosis of EC is not well known, recurrences are common even after treatment. In a pooled analysis involving 135 patients, among 59 patients who received medical treatment, 10 patients $(17.0 \%)$ showed recurrence of EC after medical treatment [7], whereas only 1 patient (2.6\%) showed recurrence after surgery among 38 patients who were treated with surgery. However, in generalized lesions, medical treatment should be accompanied by surgical treatment [4]. Therefore, to prevent recurrence, our patient was treated with antihistamines (hydroxyzine hydrochloride) for 15 months. After 15 months, CT and cystoscopy showed no recurrence, and we decided to discontinue treatment with hydroxyzine hydrochloride and closely monitor the patient with CT. The patient was followed-up for 23 months without evidence of recurrence.

In conclusion, clinicians should consider the possibility of EC in patients presenting with hematuria, fever, and 
suprapubic pain with a bladder mass. Pathologic diagnosis can be made using cystoscopic biopsy or TURB, and this is essential to distinguish malignancy. In many cases, medical treatment shows successful outcomes, but partial cystectomy can be safely performed in patients with large mass-forming EC associated with urachal cyst, without any major complications.

\section{Abbreviations \\ EC: Eosinophilic cystitis; TURB: Transurethral resection of the bladder tumor; CT: Computed tomography.}

\section{Acknowledgements}

Not applicable.

\section{Authors' contributions}

HBS wrote the initial manuscript and substantively revised it. HSP has participated in searching the prior references. JHK performed the histologic examination of the bladder. JP, corresponding author, has made contributions to the conception of the study and revised the manuscript. All authors read and approved the final manuscript.

\section{Funding}

This research was supported by the Basic Science Research Program through the National Research Foundation of Korea (NRF) funded by the Ministry of Education (2019R111A3A01060913). This funding source had no role in the design of this study and will not have any role during its execution, analyses, interpretation of the data, or writing the manuscript.

\section{Availability of data and materials}

All data generated or analysed during this study are included in this published article.

\section{Declarations}

\section{Ethics approval and consent to participate}

The study protocol was approved by the Institutional Review Board of the Eulji University Hospital (No. 2020-10-009). Written informed consent was obtained from our patient after explaining the present study.

\section{Consent for publication}

Written informed consent was obtained from the patient and the legal guardian for publication of this Case Report and any accompanying images. A copy of the written consent is available for review by the Editor of this journal.

\section{Competing interests}

The authors declare that they have no competing interests.

\section{Author details}

${ }^{1}$ Department of Urology, Eulji University Hospital, Eulji University School of Medicine, Daejeon, Korea. ${ }^{2}$ Department of Pathology, Eulji University Hospital, Eulji University School of Medicine, Daejeon, Korea. ${ }^{3}$ Department of Urology, Uijeongbu Eulji Medical Center, Eulji University School of Medicine, 712, Dongil-ro, Uijeongbu-si, Gyeonggi-do 11759, Republic of Korea.

Received: 28 November 2020 Accepted: 23 August 2021

Published online: 30 August 2021

References

1. Kilic S, Erguvan R, Ipek D, Gokce H, Gunes A, Aydin NE, et al. Eosinophilic cystitis. A rare inflammatory pathology mimicking bladder neoplasms. Urol Int. 2003;71:285-9.

2. Thomas JC, Ross JH. Eosinophilic cystitis in a child presenting with a bladder mass. J Urol. 2004;171:1654-5.

3. Popescu OE, Landas SK, Haas GP. The spectrum of eosinophilic cystitis in males: case series and literature review. Arch Pathol Lab Med. 2009;133:289-94.

4. Teegavarapu PS, Sahai A, Chandra A, Dasgupta P, Khan MS. Eosinophilic cystitis and its management. Int J Clin Pract. 2005;59:356-60.

5. Thompson RH, Dicks D, Kramer SA. Clinical manifestations and functional outcomes in children with eosinophilic cystitis. J Urol. 2005;174:2347-9.

6. Hellstrom HR, Davis BK, Shonnard JW. Eosinophilic cystitis. A study of 16 cases. Am J Clin Pathol. 1979;72:777-84.

7. van den Ouden D. Diagnosis and management of eosinophilic cystitis: a pooled analysis of 135 cases. Eur Urol. 2000;37:386-94.

8. Goodman TR, Connolly B, Taylor G. Eosinophilic cystitis following an infected urachal remnant. Pediatr Radiol. 1999;29:487-8.

9. Devasia A, Kekre NS, Date A, Pandey AP, Gopalakrishnan G. Eosinophilic cystitis — not that uncommon! Scand J Urol Nephrol. 1999;33:396-9.

10. Itano NM, Malek RS. Eosinophilic cystitis in adults. J Urol. 2001;165:805-7.

11. Sidh SM, Smith SP, Silber SB, Young JD Jr. Eosinophilic cystitis: advanced disease requiring surgical intervention. Urology. 1980;15:23-6.

12. Oh SJ, Chi JG, Lee SE. Eosinophilic cystitis caused by vesical sparganosis: a case report. J Urol. 1993;149:581-3.

13. Zhou AG, Amin A, Yates JK, Diamond DA, Tyminski MM, Badway JA, et al. Mass forming eosinophilic cystitis in pediatric patients. Urology. 2017:101:139-41.

14. Kumar S, Sharma V, Ganesamoni R, Singh S. Eosinophilic cystitis mimicking tuberculosis: an analysis of five cases with review of literature. Urol Ann. 2013:5:50-2.

15. Park H. Eosinophilic cystitis with recurrent urinary retention: case report. Res Rep Urol. 2017:9:51-3.

16. Werbrouck C, Marrannes J, Verhamme L, Steenkiste E, Laridon E, Van Holsbeeck B. Eosinophilic cystitis mimicking bladder tumor. JBR-BTR. 2014;97:375.

17. Rossanese M, Palumbo V, Sioletic S, Crestani A, Giannarini G, Ficarra V. Surgical treatment of eosinophilic cystitis in adults: a report of two cases and a literature review. Urol Int. 2019;102:122-4.

18. Li G, Cai B, Song H, Yang Z. Clinical and radiological character of eosinophilic cystitis. Int J Clin Exp Med. 2015;8:533-9.

\section{Publisher's Note}

Springer Nature remains neutral with regard to jurisdictional claims in published maps and institutional affiliations. 